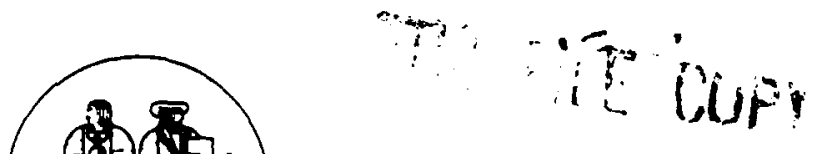

MASSACHUSETTS INSTITUTE OF TECHNOLOGY

VLSI PUBLICATIONS

VLSI Memo No. 89-538

May 1989

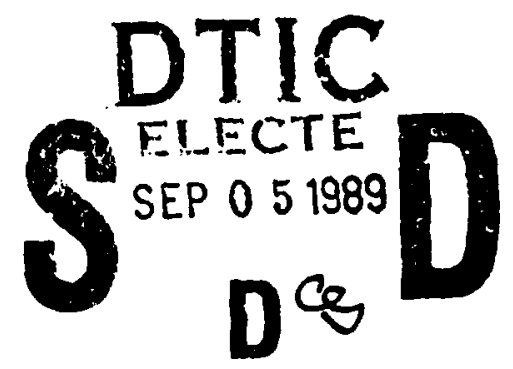

\title{
Redundancies and Don't Cares in Sequential Logic Synthesis
}

Srinivas Devadas, Hi-Keung Tony Ma, and A. Richard Newton

\section{Abstract}

The relationships between redundant logic and don't care conditions in combinational circuits are well known. Redundancies in a combinational circuit can be explicitly identified using test generation algorithms or implicitly eliminated by specifying don't cares for each gate in the combinational network and minimizing the gates, subject to the don't care conditions.

In this paper, we explore the relationships between redundant logic and don't care conditions in sequential circuits. Stuck-at faults in a sequential circuit may be testable in the combinational sense, but may be redundant because they do not alter the terminal behavior of anon-scan sequential machine. The sequential redundancies result in a faulty State Transition Graph (STG) that is equivalent to the STG of the true machine. -

We present a classification of redundant faults in sequential circuits composed of single or interacting finite state machines. For each of the different-classes of redundancies, we define don't care sets which if optimally exploited will result in the implicit elimination of any such redundancies in a given circuit. We present systematic methods for the exploitation of sequential don't cares that correspond to sequences of vectors that never appear in cascaded or interacting sequential circuits. Using these don't care sets in an optimal sequential synthesis procedure of state minimization, state assignment and combinational logic optimization result in fully testable lumped or interacting finite state machines. We present experimental results which indicate that irredundant sequential circuits can be synthesized with no area overhead and within reasonable CPU times by exploiting these don't cares.

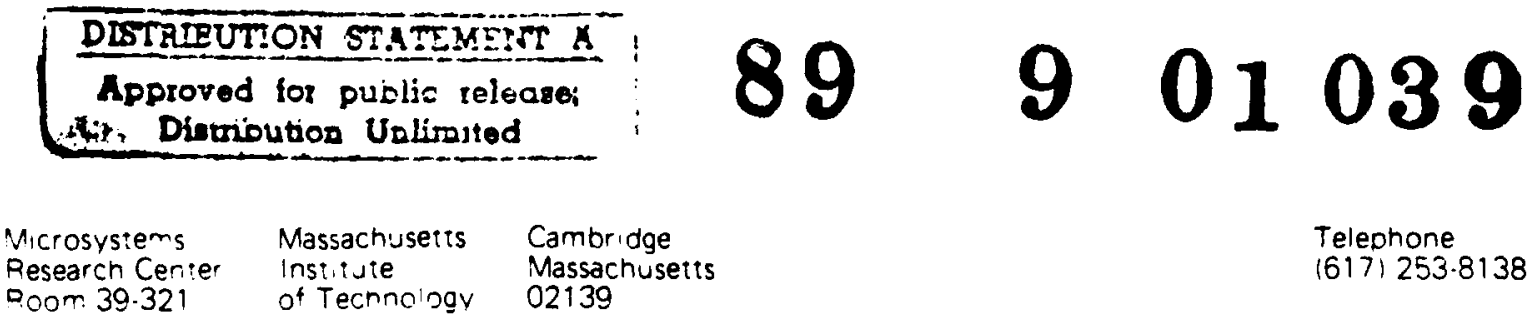




\section{Acknowledgements}

To be presented at the Intermational Test Conference, Washington, D.C., Aug. 1989. This work was supported in part by the Defense Advanced Research Projects Agency under contract number N00014-87-K-0825.

\section{Author Information}

Devadas: Department of Electrical Engineering and Computer Science, Room 36-848, MIT, Cambridge, MA 02139. (617) 253-0454.

Ma and Newton: Department of Electrical Engineering and Computer Science, University of California, Berkeley, CA 94720.

Copyright 1989 MIT. Memos in this series are for use inside MIT and are not considered to be published merely by virtue of appearing in this series. This copy is for private circulation only and may not be further copied or distributed, except for government purposes, if the paper acknowledges U. S. Government sponsorship. References to this work should be either to the published version, if any, or in the form "private communication." For information about the ideas expressed herein, contact the author directly. For information about this series, contact Microsystems Research Center, Room 39-321, MIT, Cambridge, MA 02139; (617) 253-8138. 


\title{
Redundancies and Don't Cares in Sequential Logic Synthesis
}

\author{
Srinivas Devadas \\ Department of Electrical Engineering and Computer Science \\ Massachusetts Institute of Technology, Cambridge \\ Hi-Keung Tony Ma and A. Richard Nerton \\ Department of Electrical Engineering and Computer Sciences \\ University of Californis, Berkeley
}

\begin{abstract}
The relationships between redundant logic and don't care conditions in combinational circuits are well known. Redundancies in a combinational circuit can be explicitly identified using test generation algorithms or implicitly eliminated by specifying don't cares for each gate in the combinational network and minimizing the gates, subject to the don't care conditions.

In this paper, we explore the relationships between redundant logic and don't care conditions in sequential circuits, extending the results of [5]. Stuck-at faults in a sequential circuit may be testable in the combinational sense. but may be redundant because they do not alter the terminal behavior of a non-scan sequential machine. These sequential redundan. cies result in a faulty State Transition Graph (STG) that is equivalent to the STG of the true machine.

We present a classification of redundant faults in sequential circuits composed of single or interacting finite state machines. For each of the different classes of redundancies, we define don't care sets which if optimally exploited will result in the implicit elimination of any such redundancies in a given circuit. We present systematic methods for the exploitation of sequentzal don't cares that correspond to sequences of vectors that never appear in cascaded or interacting sequential circuits. Ising these don't care sets in an optimal sequential synthesis procedure of state minimization, state assignment and combinational logic optimization results in fully testable lumped or interacting finite state machines. We present experimental results which indicate that irredundant sequential circuits can be synthesized with no area overhead and within reasonable CPI times by exploiting these don't cares.
\end{abstract}

\section{Introduction}

The connection between logic minimization and test generation for combinational circuits is well known and has been systematically investigated [2] [1]. The connection rests on the simple observation that the absence of a test is associated with redundancy in the logic network.

The sources of redundancy in combinational circuits are well understood and algorithms are available for making representations of incompletely specified combinational logic functions irredundant. The algorithms in [1] determine a don't care set for each gate in a multi-level network and minimize the logic furction corresponding to the gate, subject to these don't care conditions to produce an irredundant, fully testable circuit. Combinational test generation algorithms (e.g. [8], [11]) can be used to explicitly identify redundancies in a logic circuit, which can then be eliminated.

Redundancies in sequential machines may alter the functionality of the combinational logic in the (non-scan) sequential machine, while maintaining the terminal behavior of the machine. Thus. a stuck-at fault may be testable from a combinational point of view, but may be sequentially redundant. This redundant fault results in a faulty State Transition Graph (STG) that is equivalent to the true STG.
Sequential teat generation and sequential logic syntheais al. gorithms are much lese developed than their combinational counterparts. The observation that the abence of a teat is asoociated with redundancy holds for sequential circuits as well. While test generation algorithms can conceivably be used to produce irredundant sequential circuits, by explicitly identifying and eliminating redundancy, such an approach would require astronomical CPU time for anything but the smallest circuits, given the efficiency of state-of-the-art sequential test generation algorithms.

The relationships between don't care conditions and redundancies in sequential circuits are not well understood at present. Intuitively, one would expect that sequential redundancies are intimately related to representations of don't cares in the various steps of sequential logic synthesis, namely, state minimization, state assignment and combinational logic optimization.

In [7] and [4], constrained synthesis procedures that result in fully and easily testable finite state machines were presented. These procedures effectively involve the addition of extra logic to produce an easily testable machine. In [5], a classification of redundancies in sequential circuits composed of a single finite state machipe was presented. An optumal synthesis procedure that results in a fully testable sequential machine was given.

In the work presented in [5], each of the different classes of redundancies in single FSMs was shown to be tied to a don't care set, which if optimally exploited results in the implicit elimination of that form of redundancy. The work in [5] was restricted to single finite state machines (FSMs). Multilevel sequential circuits composed of interacting finite state machines, are common in industrial chip designs and are more complicated. The types of possible redundancies and their corresponding don't care sets in cascaded/interconnected FSMs are likewise more complicated.

We show in the cascaded machine case that sequential don't cares, corresponding to sequences of vectors that never occur, at the inputs (outputs) of the driven (driving) FSM have to be used to optimize the driven (driving) machine, in order to eliminate certain kinds of redundancies. We present systematic methods of exploiting sequential don't cares in cascaded circuits. Interacting finite atate machines can be viewed as separate occurrences of cascades and don't care sets can be iteratively used on the difierent cascades to eliminate redundancy. Finally, we present experimental results which indicate that fully testable interacting FSMs can be produced from State Transition Graph descriptions vis optimal sequential logic synthesis with reasonable CPU time expenditure.

In the next section, basic definitions and notations used are given. In Section 3 , we classify redundancies in single finite atate machines and the don't care sets required to eliminate these redundancies. We present new algorithms for the systematic exploitation of sequential don't cares in cascaded circuits in Section 4. The implicit elimination of redundancies in cascaded finite state machines is the subject of Section 5. Iterative optimization procedures for interacting FSMs are discussed in Section 6 . Preliminary experimental results are ides presented in Section 7.
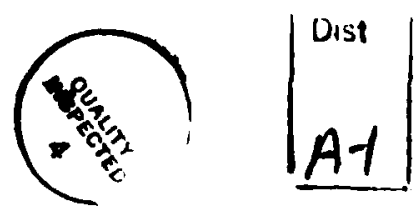


\section{Preliminaries}

A variable is a symbol representing a single coordinate of the Boolean space ( $.8 . a)$. A literal is a variable or its negation (e.g. $a$ or $\bar{d}$ ). A cube is a set $C$ of literals such that $x \in C$ implies $\bar{I} C($ e.g., $\{a, b, \bar{c}\}$ is a cube, and $\{a, \bar{a}\}$ is not a cube). A cube represents the conjunction of its literals. The trivial cubes, witten 0 and 1 , represent the Boolean functions 0 and 1 respectively. An expression is a set $f$ of cubes. For example. $\{\{a\},\{b, \bar{c}\}\}$ is an expression consisting of the two cubes $\{a\}$ and $\{b, \bar{c}\}$. An expression represents the disjunction of its cubes.

A cube may also be written as a bit vector on a set of variables with each bit position representing a distinct variable. The values taken by each bit can be 1,0 or 2 (don't care), signifying the true form, negated form and non-existence respectively of the variable corresponding to that position. A minterm is a cube with only 0 and 1 entries.

A finite state machine is represented by its State Transition Graph (S「G), $G(V, E, W(E))$ where $V$ is the set of vertices corresponding to the set of states $S$, where $\|S\|=N$ is the cardinality of the set of states of the FSM. an edge joins $v_{i}$ to $r$ if there is a primary input that causes the FSM to evolve from state $v_{i}$ to state $v_{j}$, and $W(E)$ is a set of labels attached to each erige. each label carrying the information of the value of the inpul that caused that transition and the values of the primary outputs corresponding to that transition. In general, the $H(E)$ iabels are Boolean expressions. The number of inputs and outputs are denoted $\hat{N}_{i}$ and $N_{0}$ respectively. The input combination and present state corresponding to an edge or set of e'ges is $(i, s)$. where $i$ and $s$ are cubes. The fanin of a state. $q$ is a set of edges and is denoted $f \operatorname{anin}(q)$. The fanout of a state $q$ is denoted fanout $(q)$. The output and the fanout state of an edge (i. $s) \in E$ are $o((i, s))$ and $n((i . s)) \in V$ restectivaly

Given $\therefore$ inputs to a machine. $2^{\wedge}$ ed ges with minterm input labels fan out from each state. A STG where the next state and output labels for every possible transition from every state are defired corresponds to a completely specified machine. An incompletely specified machine is one where at least one transition edge from some state is not specified.

A starting or initial state is assumed to exist for a machine, also called the reset state. Given a logic-level finite state machine with $N_{b}$ latches. $2^{N_{b}}$ possible states exist in the machine. A state which can be reached from the reset state via some inpui vector sequence is called a valid state in the STG. The input vector sequence is called the justification sequence for that state. A state for which no justification sequence exists is called an invalid state. Given a fault $F$, the State Transition Graph of the machine with the fault is denoted $G^{F}$. Two states in a State Transition Graph $G$ are equivalent if all possible input sequences when the machine is initiall in either of the two states produce the same output response

A State Transition Graph $G_{1}$ is said to be isomorphic to another State Transition Graph $G_{2}$ if and only if they are identical except for a renaming of states

The fault model assumed is single stuck-at. A finite state machine is assumed to be implemented by combinational logic and feedback registers. Tests are generated for stuck-at faults in the combinational logic part

A primitive gate in a network is prime if none of its inputs can be removed without causing the resulting circuit to be functionally different. A gate is irredundant if its removal causes the resulting circuit to be functionally different. A gatelevel circuit is said to be prime if all the gates are prime and irredundant if all the gates are irredundant. It can be shown that a gate-level circuit is prime and irredundant if and only if it is $100 \%$ testable for all single stuck-at faults.

We differentiate between two kinds of redundancies in a sequential circuit. If the effect of the fault cannot be observed at the primary outputs or the next state lines, beginning from any state. with any input vector. the fault is deemed combi-

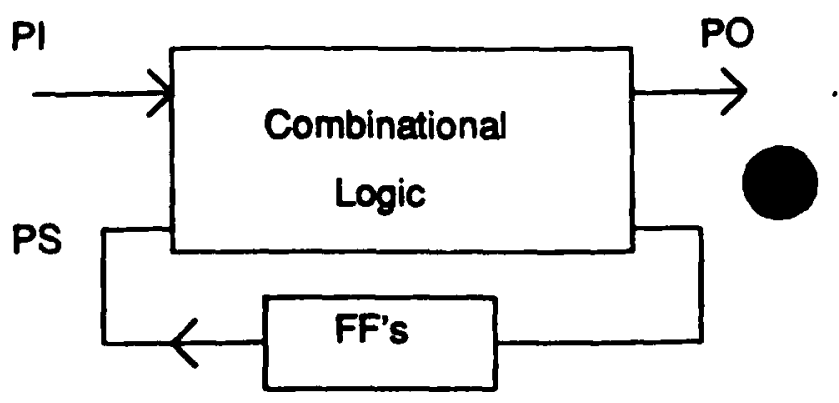

(a)

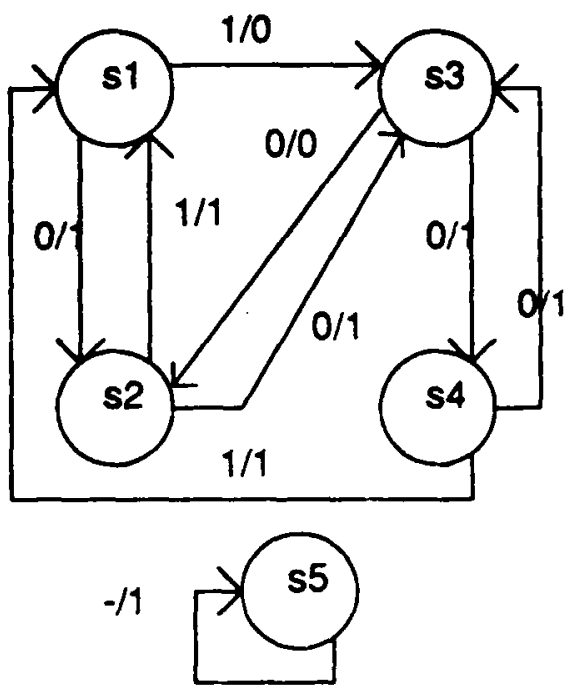

(b)

Figure 1: A Sequential Circuit

nationally redundant. A sequentially redundant fault is a fault that cannot be detected by any input sequence and is not combinationally redundant.

To detect a fault in a sequential machine, the machine has to be placed in a state which can then excite and propagate the effect of the fault to the primary outputs. The first step of reaching the state in question is called state justification. The esen $t$ step is called fault excitation-and-propagation.

A: adge in a State Transition Graph of a machine is said $t$ to orrupted by a fault if either the fanout state or outD. Del of this edge is changed because of the existence of the tault. A path in a State Transition Graph is said to be corrupted if at least one edge in the path has been corrupted.

A sequence of vectors $V S_{1}$ is said to contain another sequence $V S_{2}$ (written as $V S_{1} \supseteq V S_{2}$ ), if $V S_{2}$ appears in $V S_{1}$.

A cascade of two machines $A$ and $B$ is denoted $A \longrightarrow B$. $A$ is the driving machine and $B$ the driven machine.

\section{Redundancies in Single Finite State Machines}

A sequential circuit $S$, comprised of a single FSM is shown in Figure 1(a). The State Transition Graph corresponding to the circuit is shown in Figure 1(b).

Redundant faults in $S$ may be combinationally redunds (CRFs) or sequentially redundant (SRFs). Sequentially dundant faults can be classified in to three categories. 


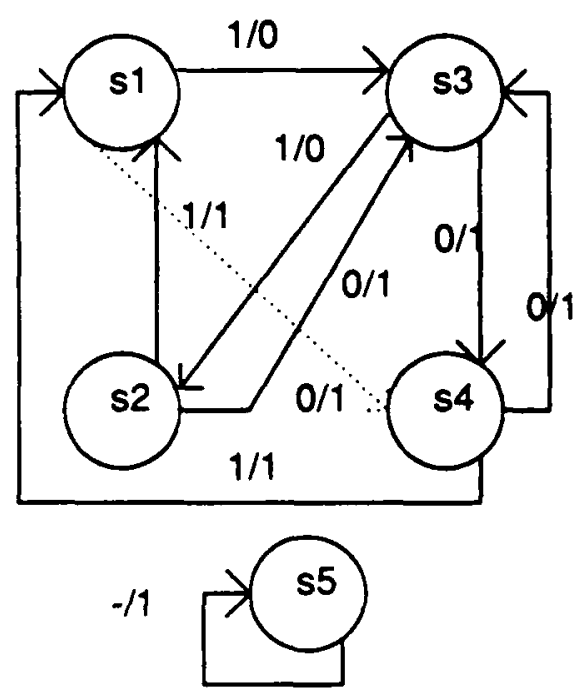

Figure 2: A Type 1 SRF

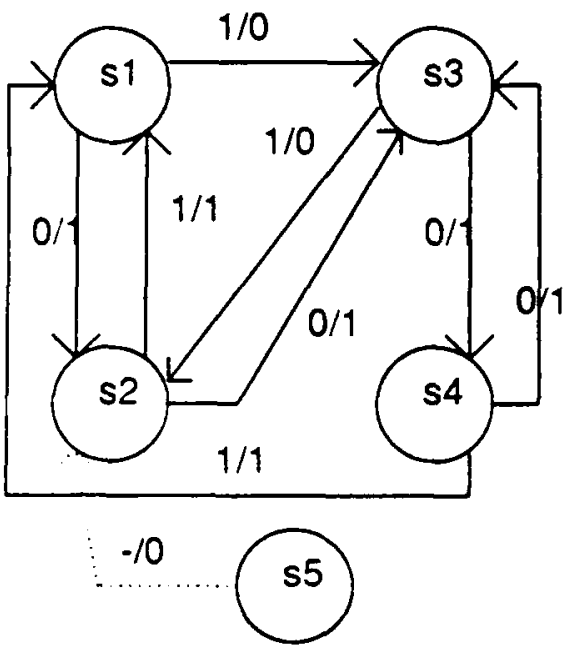

Figure 3: A Type 2 SRF

1. The fault causes the interchange/creation of equivalent states in the STG.

2. The fault does not corrupt any fanout edge of a valid state in the STG

3. The fault results in a faulty machine that is isomorphic (with a different encoding) to the original machine.

In [0]. it was shown that no other kind of sequential redundancy could exist

In Figure 1(b). states $s 2$ and $s \&$ are equivalent states. A type $1 S R F$ in $S$ may produce the faulty STG of Figure 2, where the only corrupted edge (shown in dotted lines) goes to $s 4$ instead of $s 2$ and does not change the terminal behavior of $S$. A faulty STG corresponding to a type $2 S R F$ is shown in Figure 3. Only fanout edges from an invalid state have been corrupted. In Figure 4, an isomorphic faulty machine (equivalent to the true machine) is shown where $s 2$ and $s 9$ have been interchanged.

\subsection{Eliminating Type 3 SRFs}

I. ivj. it was shown that sturk-at faults in a sequential machine implemented by a two-level combinational network could not cause isomorphism. For a sequential machine. implemented by

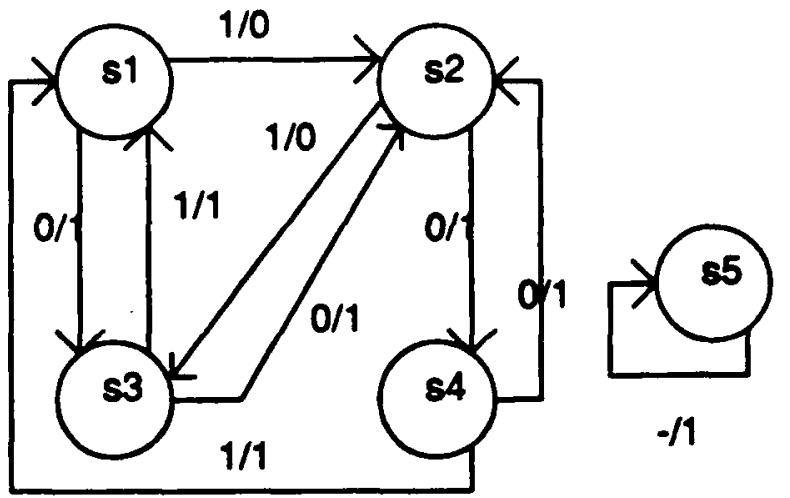

Figure 4: A Type 3 SRF

a multi-level network, stuck-at faults could conceivably produce an isomorphic faulty STG.

There are many ways of ensuring that isomorphism does not occur in multi-level networks. Isomorphism due to a fault is essentially due to a sub-optimal state assignment. The new encoding corresponding to the isomorph represents a better machine (one with the redundant line removed). A locally optimal state assignment across any given set of states can ensure that isomorphism does not occur in multi-level circuits. across this set of states. It is worthwhile to note that optimal state assignment corresponds to the optimal usage of don't cares - one does not care what the codes of the different states are so long as they are distinct.

\subsection{Eliminating Type 2 SRFs}

The codes corresponding to invalid states can be used as don't cares during logic optimization. A type 2 SRF is due to the sub-optimal usage (or no usage) of these don't cares. These redundancies will not exist if the combinational logic is made irredundant under this don't care set.

\subsection{Eliminating Type 1 SRFs}

Type $J S R F_{B}$ are related to redundant states in a sequential machine. Given a reduced machine, a fault that corrupts a single edge going to a faulty but valid state cannot be redundant, since all states are distinguishable. Thus, an initial state minimization will preclude the occurrence of the SRF of the form in Figure 2. However, we may have a case where the fault results in a faulty invalid next state that is equivalent to the true next state. This is illustrated in Figure 5. We have the true STG in Figure 5(a), that is state minimal. The invalid atate s/'s code bas been used as a don't care and $s\}$ is equivalent to state 82 after logic minimization under this don't care condition. A fault could result in the scenario shown in Figure 5(b), where a single corrupted edge whose true next state is $s 2$ produces a faulty next state, s4. The fault is redundant. This redundancy exists because we have not exploited the don't care corresponding to the edge $(0,63)$ - we can specify $n(0,83)=(s 4,82)$ and not just $s 2$. The following procedure of repeated logic minimization (modified from [5]) guarantees upon convergence that type $2 S R F_{5}$ don't exist and that single edge corrupting and certain kinds of multiple edge corrupting type $1 S R F_{s}$ don't exist. The use of extended don't cares at Step $A$ guarantees the elimination all possible of type $1 S R F \mathrm{~s}$, but these don't cares are not required in practice to produce irredundant machines.

eliminate-type1/2-SRFs $(S)$ :

t

iter $=1$;

if (iter $=1) G=\operatorname{extract-st} g(S)$; 
(a)

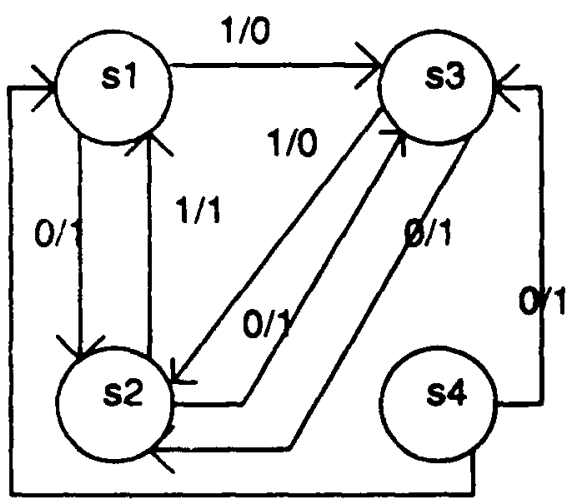

$1 / 1$

(b)

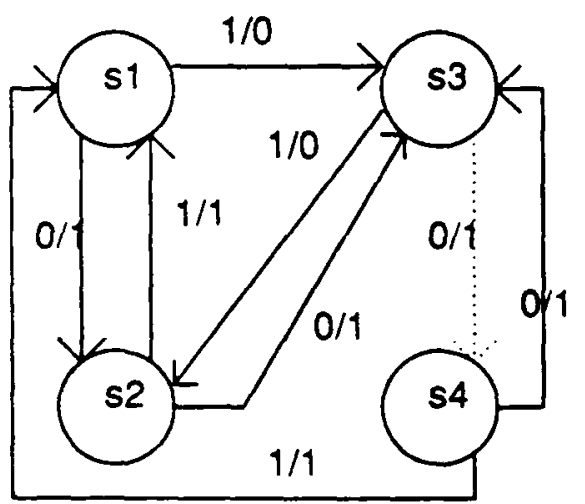

Figure 5: A Complex Type $1 \mathrm{SRF}$

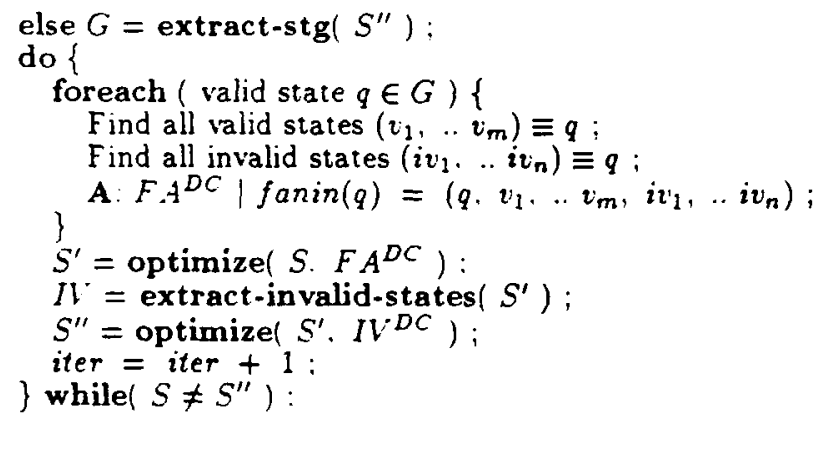

It can be proved that state minimization, a locally optimal state assignment and the procedure eliminate-type1/2SRFs() produces an irredundant sequential machine [5].

\section{Exploiting Sequential Don't Cares}

In Figure 6 . we have a machine $A$ driving another machine $B$ via a set of latches $L 1$ (We neglect $C$ for the moment). For the purposes of the discussion here, we assume that all the latches in $L 1$ are not observable. In practice, a subset of the latches may be observable.

\subsection{Don't Care Inputs for the Driven Ma- chine}

There are several don't care conditions associated with the intermediate lines corresponding to $L 1$, which are inputs to

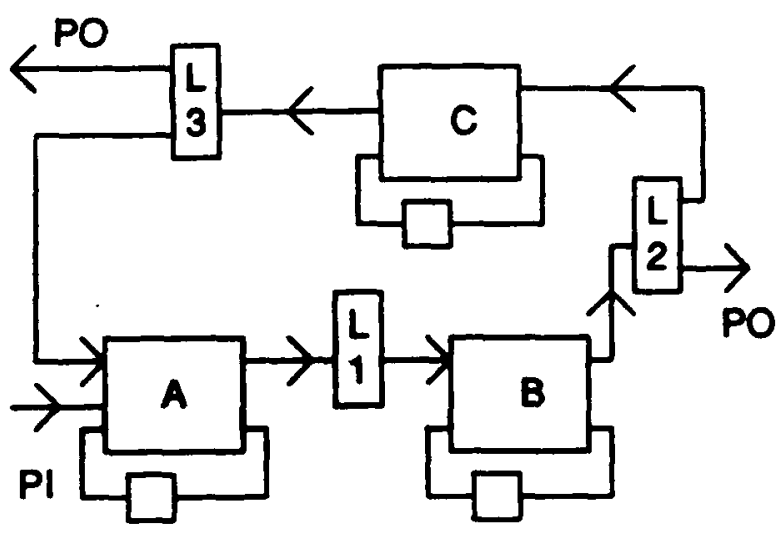

Figure 6: Interacting Finite State Machines

$B$. Let the number of intermediate/pipeline latches in $L 1$ be $N$.

1. A may or may not assert all $2^{N}$ possible output combinations. If a certain binary combination, $c_{1}$ never appears at $L 1$, then $B$ can be made incompletely specified - the transition edges corresponding to an input of $c_{1}$ need not be specified, whatever state $B$ is in (We don't care what happens when $B$ receives the input $c_{1}$ ).

2. A more general case of (1) is when a certain combination $c_{2}$ never appears at $L 1$, when $B$ is in some set of states $Q_{B} \in S_{B}$. It does appear when $B$ is in states other than $Q_{B}$. In this case, the states in $Q_{B}$ will have $c_{2}$ unspecified (If an edge on $c_{2}$ exists in $Q_{B}$, it can be removed).

3. A more complicated sequential don't care is associate with vector sequences that never appear at $L 1$, thou all $2^{N}$ separate vectors appear. $A$ does not produce alr possible output sequences. This type of don't care does not have a straightforward interpretation. Edges in the State Transition Graph of $B$ cannot be removed or left unspecified directly.

Both (1) and (2) can be easily exploited via the use of standard state minimization algorithms that handle incompletely specified machines [10]. However, exploiting the don't care input sequences is more complicated and systematic methods have not been proposed to date.

In Figure 7, a State Transition Graph corresponding to a possible $B$ machine is shown. The machine is state minimal. We assume that each transition edge in $B$ is irredundant, i.e. $B$ makes every transition with appropriate input sequences. A don't care input sequence is shown below the Graph. Such a don't care sequence implies that certain seguences of transi. tions will not be made by $B$.

A don't care input sequence is assumed to have a length greater than 1. Given a don't care sequence $D C$, all sequences $S E$ such that $S E \supseteq D C$ are also don't care eequences. We define an atomic don't care sequence as one that does not contain any other don't care sequence. Thus, any subsequence of an atomic don't care sequence is a care sequence. In the sequel, we consider only atomic don't care sequences.

Our problem lies in exploiting this form of don't care, so as to optimize $B$. In the general case, we will have a set of don't care sequences. We can state the following lemma.

Lemma 4.1 : Given a machine $B$ and a set of don't care sequences $D C_{j}, 1 \leq j \leq N_{C}$, if two states in $B, s 1$ and $s ?$ have distinguishing sequences $I_{i}, 1 \leq i \leq N_{D}$ such that $f$ each $k, I_{k} \supseteq D C_{l}$ for somel, then 81 and $s 2$ are equivalent $B$ under the $D C_{j}$. 


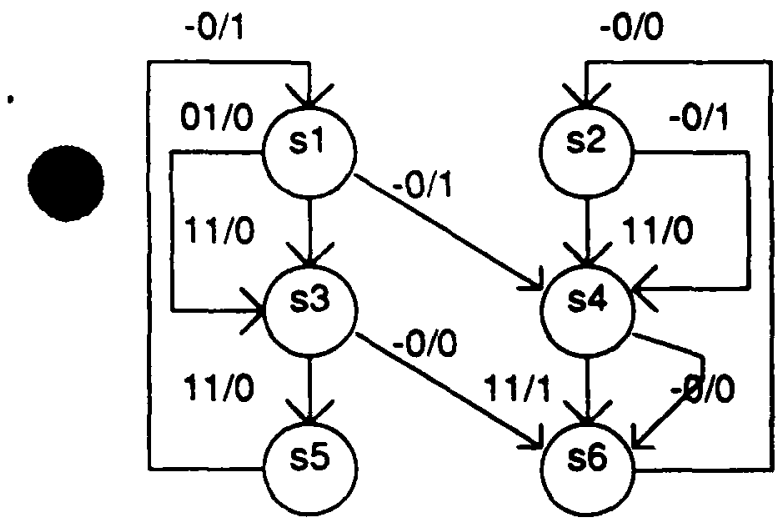

$(11,11)$

Figure 7: Input Don't Care Sequences

Proof: Since the $D C_{j}$ can never occur, it means the $I_{i}$ can never occur. Therefore. $s 1$ and $s 2$ in $B$ are equivalent under $D C_{3}$.

Q.E.D.

An approach to exploit don't cares based on Lemma 4.1 would entail producing all distinguishing seques. es for every pair of states in $B$ and checking for the containment condition. Pairs satisfying the condition can be merged. This is potentially very time consuming: a pair of states may have many distinguishing sequences and we have to find them for every possible pair. A more efficient approach is now outlined.

In this approach. given a set of don't care sequences, $B$ is transformed into a new machine $B^{\prime}$ which has a greater number of states. but is more incompletely specified than $B$. $B^{\prime}$ is siate ninimized to obtain $B^{\prime \prime}\left(\left\|S_{B^{\prime \prime}}\right\| \leq\left\|S_{B}\right\|\right)$. The pseuda-code below illustrates the procedure.

exploit-input-dc( $B . D C)$

$$
B^{\prime}=B \text { : }
$$

foreach (don't care sequence $D C_{1}$ ) \{

foreach (depth-first path $P=\epsilon_{1}, . . e_{K} \in B^{\prime}$ ) if $\left(P \supseteq D C_{1}\right)\{$

for $i=2: i \leq K: i=i+1)$

$s_{i}=e_{i}->$ fanout

make states $s_{i}^{\prime}$ and $s_{i}^{\prime \prime}$;

$\operatorname{fanin}\left(s_{i}^{\prime}\right)=e_{1-1}$

$\operatorname{fanin}\left(s_{i}^{\prime \prime}\right)=\operatorname{fanin}\left(s_{i}\right)-e_{i-1}$

if $\left(\operatorname{fanin}\left(s_{1}^{\prime \prime}\right)=\phi\right)$ delete $s_{i}^{\prime \prime}$;

if $\left(i<H^{\prime}\right)$

fanout $\left(s_{i}^{\prime}\right)=f \operatorname{anout}\left(s_{i}^{\prime \prime}\right)=\operatorname{fanout}\left(s_{i}\right) ;$ else \{

fanout $\left(s_{1}^{\prime}\right)=\operatorname{fanout}\left(s_{1}\right)-e_{i-1} ;$ \}

fanout $\left(s_{1}^{\prime}\right)=$ fanout $\left(s_{i}\right)$

delete $s_{2}$ :

\}$_{B^{\prime \prime}}^{\}^{\prime}}=$ state-minimize $\left(B^{\prime}\right)$;

The procedure is effectively producing a machine where the don't care sequences are not specified. but otherwise has the same functionality as the original machine. This means that if any two states in $B$ satisfy the conditions of Lemma 4.1, these two states will not possess a distinguishing sequence in $B^{\prime}$ and will thus be compatible during state minimization. A smalier machine $B^{\prime \prime}$ will be obtained after state minimization.

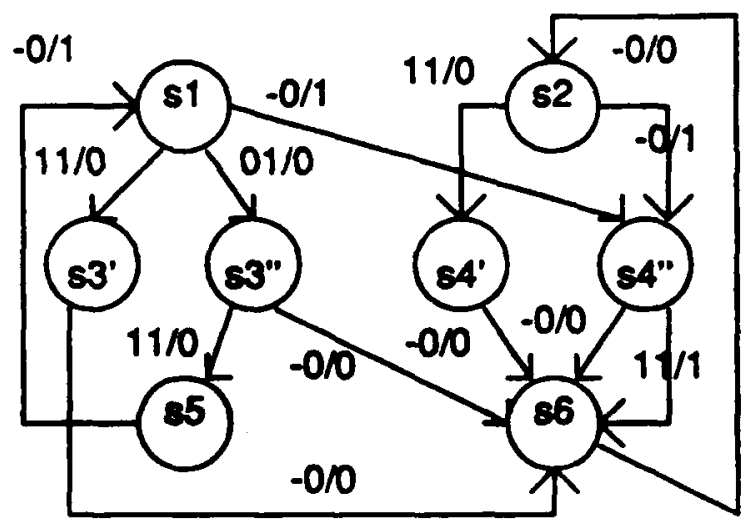

Figure 8: Expanding the Original Machine

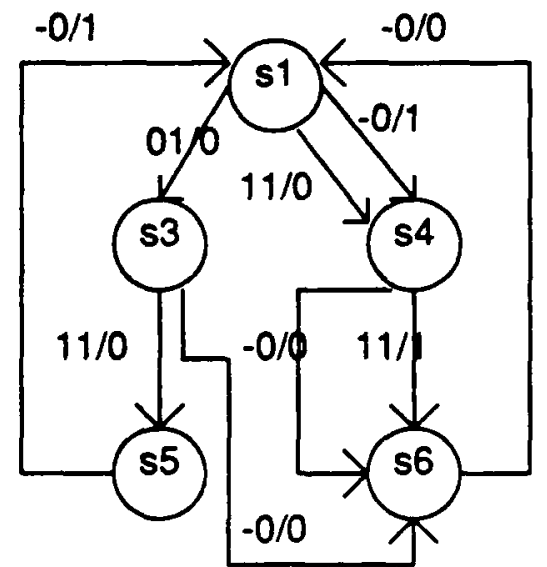

Figure 9: State minimization after Expansion

When $i=p<K$ in the for loop above, the fanout of $s_{p}$ is duplicated for the states $s_{p}^{\prime}$ and $s_{p}^{\prime \prime}$ - the edge $e_{p}$ is also duplicated. Hence, at the next iteration, one of the $e_{p}$ fans into $s_{p+1}^{\prime}$ and the other $e_{p}$ (as well as the remaining fanout edges from $s_{p}^{\prime}$ and $s_{p}^{\prime \prime}$ ) into $s_{p+1}^{\prime \prime}$.

An illustrative example is given in Figures 8 and 9 . Th: machine and the don't care sequence of figure 7 produce ar: expanded machine, shown in Figure 8. State minimizing this machine produces the result of Figure 9, which has one less state than the original machine of Figure 7.

Given a cascade, we need to generate the set of sequences that the driving machine in a cascade $A \longrightarrow B$ never asserts, 80 as to optimize the driven machine $B$ as in Figures 8 and 9. This is done by generating don't care sequences of increasing length, beginning from a length of 2 . Starting from each possible state in $A$, all possible 2-vector sequences are found. Single vectors that don't occur are added to this set. and the set is "complemented" to find the atomic 2-vector sequences that don't occur. Next, all sequences of length 3 that $A$ asserts are found. The single-vector and 2-vector don't care sequences are added to this set and the union is complemented to find the atomic don't care sequences of length 3 .

\subsection{Don't Care Outputs for the Driving Ma- chine}

The sequential don't cares discussed thus far are a product of the constrained controllability of the driven machine $B$ in cascade $A \longrightarrow B$. There is another type of don't care due to the constrained observability of the driving machine $A$. We 


\begin{tabular}{|c|c|c|c|c|c|}
\hline i) sa1 & sa2 & INT1 & INT1 & qb1 & $q b^{2}$ \\
\hline i2 sa 1 & sa3 & INT2 & INT2 & $q b 1$ & qb3 \\
\hline sa2 & sa1 & INT2 & INT1 & $a b 2$ & $q b 2$ \\
\hline 12 & sa3 & INT1 & INT2 & b2 & $q b 2$ \\
\hline$i 1 s$ & say & INT1 & INT1 & b3 & $q b 2$ \\
\hline i2s & sa2 & INT1 & INT2 & ab3 & qb3 \\
\hline
\end{tabular}

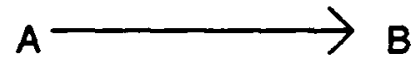

Figure 10. Output Expansion

focus on the individually state minimized tables of Figure 10. The intermediate inputs/outputs have been given symbolic codes. Given that $A$ feeds into $B$, it is quite possible that for some transition edge $\epsilon_{1} \in A$. it does not matter if the output asserted by this particular transition edge is, say, INTi or $I N T j$. In fact. in Figure 10 , the 3rd transition edge can be either $I N T 1$ or $I N T 2$. without changing the terminal behavior of $A \longrightarrow B$. This is a don't care condition on $A$ 's outputs. It is quite possible that making use of these don't cares can reduce the number of states in $A$. In fact, if one replaced the output of the 3 rd edge in $A$ (Figure 10) by $I N T 1$ instead of $I N T 2$, we would obtain one less state after state minimization. (sa2 becornes equivalent to sa9).

Given a cascade $A \longrightarrow B$. we give below a systematic procedure to detect this type of don't care. i.e. expand the output of each transition edge of $A$ to the set of all possible values that it :an take while maintaining the terminal behavior of A - B. Standard state minimization procedures can exploit don t care outputs. represented as cubes. However, state minimization procedures have to be modified in order to exploit iransition edge outputs represented as arbitrary Boolean expressions (multiple cubes).

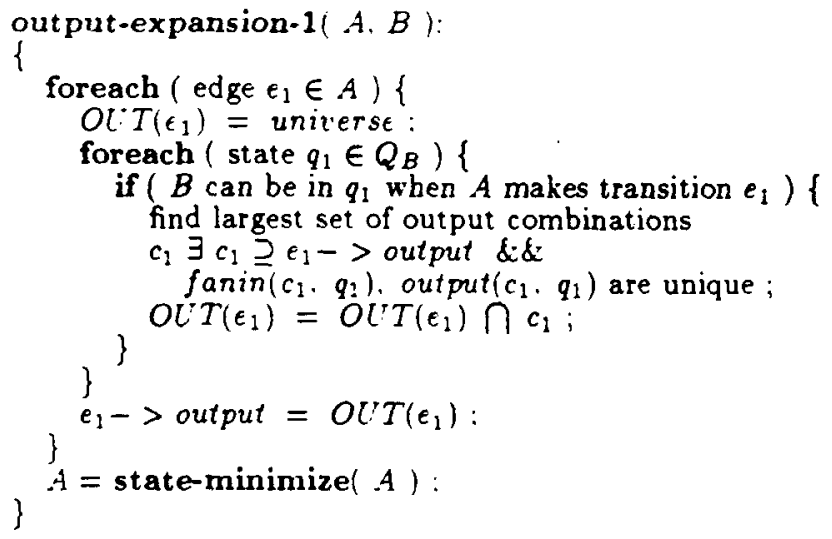

A transition edge $e_{1}$ in $A$ is picked. The set of states that $B$ can be in when $A$ makes this transition is found. Given this set of states. the largest cube (or set of output combinations) that covers the output of the edge and produces a unique next st ate and a unique out put when $B$ is in any one of the possible states is found (corresponds to OUT(e $\left.e_{1}\right)$ ). The output of $e_{1}$ is expanded to the cube. The process is repeated for all edges in $A$

The state minimization procedure proposed in $[10]$ can be used for incompletely specified finite state machines. However. after output expansion. we may have a multiple-output FSM in which a transition edge has an output that can belong to a subset of symbolic or binary values. rather than the universe of possible values (as in the incompletely specified case)

In the state minimization procedure of $[10]$, two states are deemed to be compatible if the output combinations that can be asserted by each pair of corresponding fanout edges of the two states intersect. One can envision a situation
Where the possible output combinations of the fanout wges of $q_{1}, q_{2} \in S_{M}$ intersect leading to a compatibility relation $q_{1} \rightarrow q_{2}$, with aimilar compatibility relation $q_{2} \rightarrow q_{3}$ and $q_{1} \rightarrow q_{s}$. However, the three-way intersection between the possible output combinations of the fanout edges of $q_{1}, q_{2} \mathrm{an}$ $q_{3}$ may be a null intersection, implying that $q_{1}, q_{2}$ and $q_{3} \mathrm{ca}$ not be merged into a single tate, even though alf the required pairwise compatibility relations exist. In the binary-valued output case, if the posible output combinations can be represented as a single cube, then such a situstion will not occur, since the three-way internection of a set of three cubes bas to be non-empty if the pairvive interwections are non-empty. But, in the case of multiple cubes or Boolean expreasions specifying the output combinations for fanout ed ges, an additional check has to be performed during state minimisation during the selection of the compatibility pairs to see if three or more sets of states can, in fact, be merged, preserving functionality.

\section{Fully Testable Cascaded Finite State Machines}

In this section, we present a classification of redundant faults in a cascade. We define don't care sets tied to each of these forms of redundancy and give a synthesis procedure that pro duces an irredundant cascade.

\subsection{Redundancies in a Cascade}

Redundancies in a cascade $A \longrightarrow B$ can be classified into four categories. The intermediate lines are denoted INT.

1. $F \in A$ that cannot propagate to the intermediate lines $I N T$.

2. $F \in A$ that propagates to $I N T$ but not $P O$, the primary outputs of $B$.

3. $F \in B$ that does not propagate to $P O$, but would have $I N T$ were completely controllable.

4. $F \in B$ that does not propagate to $P O$ and would not have even if INT were completely controllable.

Obviously, there can be no other class of redundancy.

It is easy to see that redundancies of type (1) and (4) are associated with the single machines $A$ and $B$. If $A$ and $B$ are irredundant, these redundancies will not appear in a cascade $A \longrightarrow B$. It is convenient to further classify the redundancies of type (2) and (3).

2. Redundancies of type 2 can be classified into

(a) $F \in A$ produces a int $F \neq$ int (a faulty output not equal to the true output) that is a specified output for all states that $B$ can be in. int $F$ elicits the same response as int from $B$ for all of these states and therefore $F$ is redundant.

(b) $F \in A$ produces an unspecified or invalid output int $^{F}$ for the states $B$ can be in and elicits the same response from $B$. In this case, one may elicit the same response from $B$ or $B$ might be moved to an invalid state that is equivalent to the true state resulting in redundancy.

(c) A more complicated case of $2(\mathrm{a}) /(\mathrm{b})$, where $A$ produces a sequence of faulty outputs int $F$,.. int $N^{F}$ instead of int 1 ,... int $N$, such that the first output moves $B$ into an invalid state that is not equivalent to the true state, but this state effectively becomes equivalent to the true state due to int $2^{F}$,..int $N^{F}$

3. Redundancies of type 3 can be classified into 
(a) $F \in B$ requires a transition edge in $B$ that cannot be justified for excitation/propagation to the primary output or next state lines.

(b) A transition edge that propagates $F \in B$ to the next state lines exists and the faulty state produced is a valid state. The faulty fault-free stale pair in $B$ possess a distinguishing sequence (which constitutes part of a test sequence), but this sequence cannot be produced at the outputs of $A$.

(c) Same as above, except that the faulty state that is produced is an invalid state.

Redundancies 2(a) and 2(b) are associated with single-vector don't care outputs of $A$. Of course, one may have multiple occurrences of faulty output vectors producing the same responses for a fault $F$ of type 2(a) or 2(b). Redundancy 2(c) is associated with don't care output sequences (multiple vectors) of $A$. Redundancy $3(\mathrm{a})$ is associated the simple form of input don't care described in Section 3.1, where transition edges in $B$ need not be specified. Redundancies 3(b) and 3(c) are associated with don't care input sequences to $B$.

\subsection{A Synthesis Procedure for Irredundant Cascaded Machines}

The procedure presented below represents a one-pass optimization for a cascade and eliminates a large number of redundancies in a cascade.

optimize-cascade $(A, B)$ :

output-expansion-1 $(A, B)$

irredundant -1 ( .4$)$

exploit-input-dc $\left(B, D C^{A}\right)$;

irredundant $-1(B)$ :

Don't care outputs of $A$ and don't care inputs to $B$ are exploited. The procedure irredundant-1() uses the techniques described in the previous section to make a single machine irredundant in isolation.

Theorem 5.1 The procedure optimize-cascade() produces a cascade $A-B$ that is irredundant for all type 1 , type :(a). type $3(a)$. typ: $9(b)$ and type 4 faults

Proof: Type 1 and type 4 faults cannot exist, since $A$ and $B$ are irredundant in isolation.

After the procedure exploit-input-dc() has been used, each remaining (specified) edge in the machine $B$. can be justified. by some in put sequence to $A$. After $B$ has been made prime and irredundant. we are guaranteed that at least one of the originally specified edges is a test vector in the combinational sense for any fault $F \in B$. That is, we have a vector that excites and propagates $F$ to the primary outputs of $B$ or the next state lines. This vector can be reached controlling $A$ alone. Therefore. $F$ cannot be a redundancy of type $3(a)$.

Next. consider redundancies of type 3(b). After the procedure exploit-input-dc() has been used on $B$ with a complete don ' care input sequence set. each pair of valid states remaining in $B$ possess a distinguishing sequence that is not in the don't care input sequence set. This means that each pair of valid states can be distinguished via an input sequence to $A$ Therefore. if $F \in B$ produces a faulty fault-free state pair such that the faulty state is a valid state, then we have a distinguishing (test) sequence for $F$ and $F$ cannot be a redundancy of type 3 (b). However. the same cannot be said of faulty states that are invalid and $F$ may be a redundancy of type $3(c)$.

Redundancies of type 2(a) cannot exist because output expansion has been performed on $A$. using output-expansion1i). A fault $F \in A$ can be initially propagated to the outputs of $A$ or the next atate lines. If all teat vectors for $F$ propegate $F$ to the output lines alone and produce valid/opecified foulty outputs (if even one vector produces as invalid output, $F$ cannot be redundancy of type 2(a)), then because we have exploited the output don't cares for each transition odge in $A$, we are guaranteed that at least one of the vectors (edges) corrupted by $F$ will elicit a different response for tome tate that $B$ can be in. (By different response we mean that $B$ goes to a different atate or produce a different output). On the other hand, if $F$ is propegated to the next ante limed alone, then a corrupted vector will exiot such that it produces a fulty otate that can be dintinguished from the true otate under the outpat don's care set. Thi means we have a diftinguibhing input vector sequence (to $A$ ) such that the final falty ontput necessarily elicits a difierent response from $B$ or is an invalid/unspecified output. If $F$ is propagated to both the outputs and the next state lines then for some test vector either the faulty output will directly elicit a different response from $B$ or the faulty fault-free state pair will possess a distinguishing sequence that eventually elicite a different response from $B$. Thus, $F$ is testable or is not a redundancy of type 2(a).

Q.E.D.

Eliminating type $1 S R F s$ in a single machine required it erative optimization due to the existence of invalid states. In a cascade, we have a similar situation where eliminating type $2(\mathrm{~b})$ and $3(\mathrm{c})$ redundancies (which might result in $B$ moving to an invalid state) requires a two-pass optimization. This is because expanding the outputs of $A$ to include invalid/unspecified outputs may introduce additional don't care input sequences to $B$.

irredundant-cascade $(A, B)$ :

f

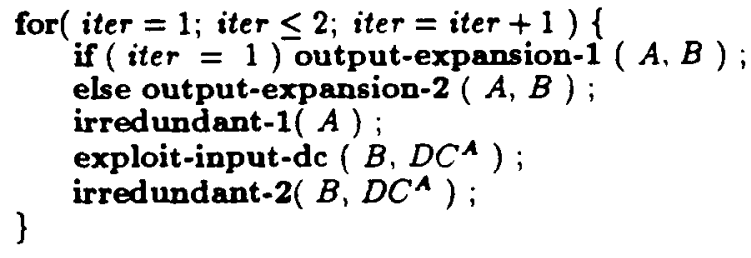

The procedure output-expansion-2() is an enhanced version of output-expansion-1(). There are two enhancements corresponding to the don't cares for type 2(b) and type 2(c) redundant faults.

1. Given an optimized $B$, for each valid state, all the invalid states that are equivalent to this state are found. We might have a situation where for a particular transition edge in $A$, an output different from the edge's output places $B$ in an invalid state that is equivalent to the true valid state. This output represents a don't care for the transition edge and is detected in output-expansion-2() (but not in output-expansion-1()). We also have the simpler situation of $A$ producing a faulty output that was originally unspecified for the state(s) $B$ is in, eliciting the same response from $B$. The output of the transition edge can be expanded to this unspecified combination.

2. Don't care output sequences are detected for $A$. The detection of these sequences is performed by checking if invalid states in $B$, that are not equivalent to valid states and reached by unspecified outputs from $A$, produce the same response in $B$ due to the corruption of other transition edges in $A$. The corrupted outputs represent a don't care output sequence for edges in $A$. A 2-vector don't care sequence is shown below.

$$
\begin{gathered}
\left(o\left(e_{1}\right), o\left(e_{2}\right)\right)=\left(e_{1}->o p, e_{2}->o p\right) \bigvee \\
\left(e_{1}->o p^{F}, e_{2}->o p^{F}\right) \quad \text { (1) }
\end{gathered}
$$


Current lubic minimizers are restricted in their capability to exploit don't cares. Don't care output sequences of the form of Eq. 1 cannot be optimally exploited, other than by exhaustive search. Fortunately, these don't cares are not required in practice - we have not encountered a single occurrence of a type $2(c)$ redundancy in a cascade, even if only single-vector don't care outputs have been used.

The $f$-oced ure irredundant-2() is also an enhancement on procedure irredundant-1().

Irredundant-2() uses eliminate-type1/2-SRFs() with an additional don't care set at Step $\mathbf{A}$. At Step $\mathbf{A}$, we have

$\mathbf{A}: F A^{D C} \mid \operatorname{fanin}(q)=\left(q, v_{1}, . . v_{m}, i v_{1}, . . i v_{n}, n i_{1}, \ldots n i_{l}\right)$

where $v_{1}, \ldots v_{m}$ and $i v_{1}, \ldots, i v_{n}$ are valid sod invalid states respectively, that are equivalent to $q$ when $B$ is viewed in isolation, i.e. deemed completely controllable. $n i_{1}, . . n i_{1}$ are states not equivalent to $q$ when $B$ is viewed in isolation, but equivalent to $q$ under the don't care set $D C^{A}$

Theorem 5.2 The procedure irredundant-cascade() results in an irtedundant cascade.

Proof: The procedure irredundant-cascade() is an enhanced version of the procedure optimize-cascade() and the arguments that type 1, type 2(a), type 3(a), type $3(b)$ and type 4 faults are testable hold here as well. We focus on possible redundancies of types $2(\mathrm{~b}), 2(\mathrm{c})$ and $3(\mathrm{c})$.

The procedure output-expansion-2() uses the additional don't care outputs for $A$ corresponding to the invalid states in $B$ that are equivalent to valid states and which are reached by outputs other than the transition edge outputs of $A$. Using these don't cares ensures that type 2(b) redundancies don't exist. The argument is similar to the argument of Theorem 5.1 for the type 2(a) redundancy. A fault $F \in A$ will immediately or eventually produce an invalid/unspecified output such tha the invalid output elicits a different response from $B$. If $B$ is moved to a faulty invalid state we are guaranteed that the invalid state is not equivalent to the true state. Thus. $F$ is testable or is not a redundancy of type $2(b)$.

Redundancies of type 2(c) are associated with don't care output sequences for $A$. That is, it does not matter if $A$ asserts one particular sequence or another due to its constrained observability. If the don't care sequences corresponding to Eq. 1 are exploited in the out put expansion procedure, we are guaranteed that the corrupted sequence does not elicit the same response as the true one from $B$

Finally: we consider redundancies of type $3(\mathrm{c})$. The additional don't care set at Step $\mathbf{A}$ in eliminate-type1/2. SRFs() will guarantee, after $B$ has been made prime and irredundant, that any faulty faulty-free state pair that is produced due to a fault $F$, regardless of whether the faulty state is valid or invalid. will possess a distinguishing sequence not in $D C^{A}$. This means that the pair can be distinguished from the inputs of $A$ and $F$ cannot be a redundancy of type $3(c)$. Q.E.D.

\section{Fully Testable Interacting Finite State Machines}

Interacting finite state machines are common in industrial chip designs. In Figure 6, an example sequential circuit composed of three interacting finite state machines was shown. In this section. we describe iterative optimization strategies for the synthesis of irredundant interacting finite state machines.

The don't care sets associated with a set of interacting FSMs are essentially the same as those in a cascade. At any given set of intermediate lines or latches that are not observable/controllable we have don't care input and output sequences. We can view an arbitrary set of interacting machines as several occurrences of individual cascades and use the don't

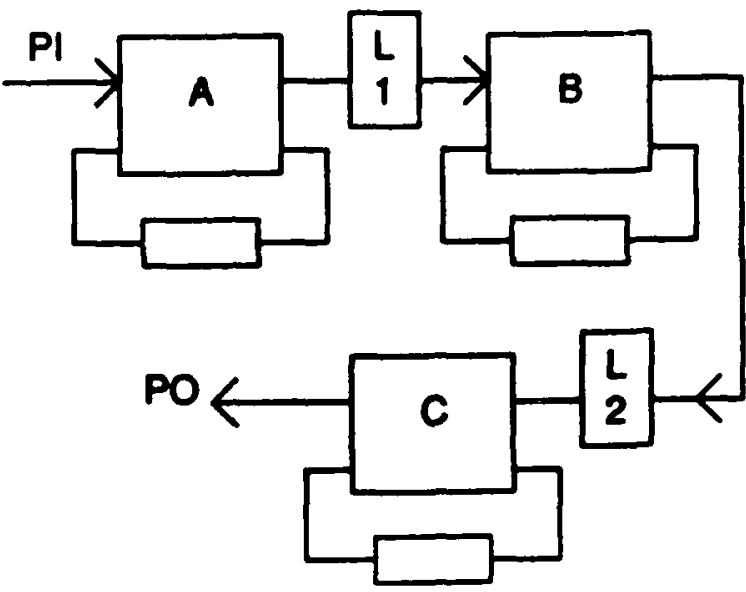

Figure 11: A Cascade Chain

care sets required for synthesizing irredundant cascades iteratively to eliminate all redundancies in the circuit.

We first focus on the cascade chain of Figure 11. There are two individual cascades corresponding to $A \longrightarrow B$ and $B \longrightarrow$ $C$. It is easy to see that if one optimizes $A \longrightarrow B$ first and then $B \longrightarrow C$, then we obtain the entire set of don't care input sequences to $C$, but not vice versa. This is because optimizing $B$ with its don't care input sequences may produce additional don't care input sequences for $C$. Thus, an appropriate order of optimization of individual cascades is required. However, optimizing $A \longrightarrow B$ before $B \longrightarrow C$ may result in missing some don't care outputs for output expansion, as illustrated in Figure 12. We have fragments of the State Transition Graphs corresponding to $A, B$ and $C$ in Figure 12 . We would raise the outputs of the edge in $A$ only if we optimized $B$ before $A \longrightarrow B$.

We have thus a conflict between the order of optimization or the individual cascades. if we wish to make use of all the don't care sets. This conflict is resolved in the cascade chain case quite simply, by an optimization $A \longrightarrow B, B \longrightarrow C$ and $A \longrightarrow$ $B$. The case of Figure 6 , a more general case where global feedback exists, is more complicated and requires iteration to convergence of the three individual cascades, $A \longrightarrow B, B \longrightarrow$ $C, C \rightarrow A$. Iteration to convergence is required because the global feedback may result in, say additional don't care inputs to $B$ after the dor $t$ care inputs to $A$ have been exploited, which in turn are dependent on the output don't cares of $C$ and 80 on.

Given an arbitrary interconnection of FSMs, the elimination of all redundancies entails the optimization of every path from the primary inputs to the primary outputs using the input don't are sets. Similarly, every reverse path from the primary outputs to the primary inputs has to be optimized for output don't cares. Iterative optimization to convergence is required in the case of feedback paths. If this is done and the machines are all irredundant in isolation, the interconnection will be irredundant. Any fault, $F$, in any machine $M$, will possess a test sequence at $M$ 's inputs whose effects can be observed at M's outputs. Exploiting don't care input sequences from the primary inputs outward to the primary outputs ensures that this test sequence can be produced at the inputs of $M$. Exploiting the don't care outputs from the primary outputs inward to the primary inputs ensures that the effect of the test sequence will be propagated to the primary outputs.

\section{Results}

In this section, we present some preliminary results obtained. using the synthesis procedures described in Section 5 and 6. 


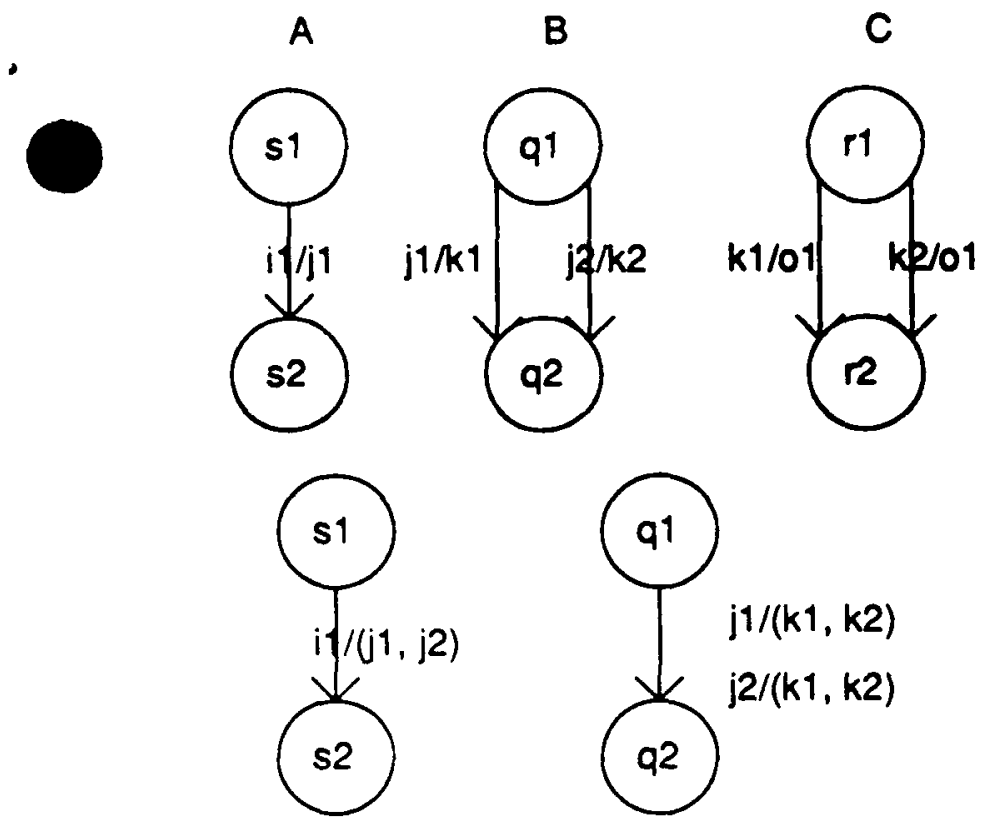

Figure 12: Order of Optimization for Don't Care Outputs

\begin{tabular}{|c|c|c|c|c|c|c|c|c|c|}
\hline \multirow[t]{2}{*}{$E x$} & \multirow[t]{2}{*}{ pi } & \multirow[t]{2}{*}{ po } & \multirow[t]{2}{*}{$\mathrm{ma}$} & \multirow[t]{2}{*}{ iI } & \multirow[t]{2}{*}{ Ta } & \multicolumn{3}{|c|}{ \#states } & \multirow[t]{2}{*}{ \#lit } \\
\hline & & & & & & MT & $\mathrm{Mr}$ & M3 & \\
\hline$f x$ & 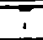 & TI & 2 & 14 & 25 & 20 & 48 & - & 704 \\
\hline$e \times 2$ & $\Pi$ & 9 & 2 & 8 & 16 & $\pi$ & 16 & & 338 \\
\hline 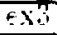 & 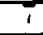 & 2 & 2 & 4 & 12 & 16 & 16 & & 186 \\
\hline$e \mathrm{Xi}_{i}$ & $:$ & $T_{1}$ & $\overline{3}$ & 13 & 28 & 20 & 32 & 19 & 926 \\
\hline exis & 5 & 21 & 3 & T!! & $3 !$ & 20 & 16 & 48 & 772 \\
\hline
\end{tabular}

Table 1: Statistics of Examples

Intensive optimization is necessary to obtain fully testable designs. If this optimization can be carried out, then the synthesized machine(s) will occupy minimal area. There is no area/performance overhead associated with this procedure. However. the CPL time requirements have to be evaluated.

iVe chose some examples in the MCNC 1987 Logic Synthesis Workshop as test cases, whose statistics are given in Table 1. Tnese machines were interconnected in various ways. In Table 1, the number of primary inputs (pi) and primary outputs (po). the number of separate machines (ma) and the number of states in each machine in the circuit (\#states) are indicated for each example. The number of intermediate, nonobservable/non-controllable lines (il) and the total number of literals (\#lit) after state assignment using MUSTANG [6] and multi-level combinational optimization using MIS [3] are also given. The total number of latches (la) corresponds to a minimum bit encoding for each machine and the pipeline latches 111 the intermediate lines

The program STALLION $[9]$ was used initially to generate tests for the original circuits. The results are given under the column ORIGINAL. The time in CPU minutes required for test generation (TPG time), the fault coverage obtained by STALLION (fcov). and the original literal count for each circuit (lit) are indicated. The circuits were optimized using the various don t cares described in the previous sections. The $C P C^{\circ}$ time required for logic optimization (logic time), test generation (TPG time), fault coverage obtained by STALLION (fcov) and the final literal counts (lit) are indicated under the column OPTIMIZE for each circuit.

All the circuits have been reduced in complexity and made

\begin{tabular}{|c|c|c|c|c|c|c|c|}
\hline \multirow[t]{2}{*}{ Ex } & \multicolumn{3}{|c|}{ ORIGINAL } & \multicolumn{4}{|c|}{ OPTLMLEE } \\
\hline & $\begin{array}{l}\text { TPG } \\
\text { time }\end{array}$ & ICOV & IIt & $\begin{array}{l}\text { Togic } \\
\text { time }\end{array}$ & $\begin{array}{l}\text { TPG } \\
\text { time }\end{array}$ & TCOV & Iit \\
\hline exI & $18.1 \mathrm{~m}$ & 95.I & 704 & $42 m$ & $77.4 \mathrm{~m}$ & 200 & 641 \\
\hline ex2 & $3.5 \mathrm{~m}$ & 91.6 & 338 & $\mathrm{Thm}$ & $4.6 \mathrm{~m}$ & 100 & 241 \\
\hline$e \times 3$ & $4.6 \mathrm{~m}$ & 95.7 & 186 & IIm & $9.7 \mathrm{~m}$ & 100 & 151 \\
\hline$e \times 4$ & 21.2m & 91.4 & 926 & $75 m$ & $18.3 \mathrm{~m}$ & 100 & 781 \\
\hline$e x 5$ & 25.1m & 92.5 & 772 & $36 \mathrm{~m}$ & $22.4 \mathrm{~m}$ & 100 & 64 \\
\hline
\end{tabular}

Toble 2: Reoult uning Don't Care Exploitation Algorithms

irredundant. STALLION eitber aborted or identified the undetected faults in the original circuits to be redundant. While the CPU times required for logic optimization are typically larger than the teat generation times, the alternative of explicitly identifying redundancies in the original circuits via test generation would expend considerably more CPU time. Redundant lines corresponding to redundant stuck-at faults can only be removed one at a time. Futhermore, removing a redundant line may introduce new redundancies and so all faults have to be checked for redundancy on each removal. Thus, the implicit elimination of redundancies via the use of don't care sets represents a much more efficient approach to the synthesis of irredundant interacting sequential machines.

\section{Conclusions}

In this paper, we explored the relationships between redundant logic and don't care conditions in sequential circuits. Redundancies in non-scan sequential circuits may be testable from a combinational viewpoint. but may produce a faulty. State Transition Graph (STG) that is equivalent to the STG of the true machine.

We presented a classification of redundant faults in sequential circuits composed of single or interacting finite state machines. Don't care sets can be defined for each class of redundancy and optimally exploiting these don't care conditions results in the implicit elimination of any such redundancies in a given circuit. In cascaded and interconnected sequential circuits, sequential don't cares are required to eliminate redundancies.

We presented preliminary experimental results which indicate that medium-sized irredundant sequential circuits can be synthesized with no area overbead and within reasonable $C P U$ times by exploiting these don't cares.

\section{Acknowledgements}

The interesting discussions with Pranav Ashar, Robert Brayton, Kurt Keutzer and Alberto Sangiovanni-Vincentelli on sequential machine testability are acknowledged. This work was supported in part by the the Defense Advanced Research Projects Agency unde: contract N00014-87-K-0825.

\section{References}

[1] K. Bartlett, R. K. Brayton, G. D. Hachtel, R. M. Jacoby, C. R. Morrison, R. L. Rudell, A. Sangiovannj-Vincentelli, and $A$. R. Wang. Multi-level logic minimization using implicit don't cares. In IEEE Transections on CAD. pages 723-740, June 1988.

[2] D. Brand. Redundancy and don't cares in logic synthesis. In IEEE Transactions on Computers, pages $947-952$, October 1883. 
[3] R. Brayton, R. Rudell. A. Sangiovanni-Vincentelli, and A. Wang. Mis: a multiple-level logic optimization system. In $I E E E$. Transartions on CAD, pages 1062-1081, November 1987

(4) S. Devadas, H-K. T. Ma, and A. R. Newton. Easily Testable PLA-ba \& Finite State Machines. In Proc. of $19 t h$ Fault Tolerant Computing Symposium, June 1989.

[5] S. Devadas, H-K. T. Ma, A. R. Newton, and A. Sangiovanni-Vincentelli. Irredundant sequential machines via optimal logic synthesis. In Electronics Research Laboratory Memorandum $M 88 / 52$, University of California, Berkeley, August 1988.

[6] S. Devadas, H-K. T. Ma, A. R. Newton, and A Sangiovanni-Vincentelli. Mustang: state assignment of finite state machines targeting multi-level logic implementations. In IEEE Transactions on CAD, pages 1290-1300, December 1988 .

$\because$ S. Devadas, H-K. T. Ma. A. R. Newton, and A. Sangiovanni-Vincentelli. Synthesis and optimization procedures for fully and easily testable nequential machines. In Proc. of International Test Conference, pages 621-630. September 1988

[0. P. Goel An implicit enumeration algorithm to generate tests for combinational logic circuits. In IEEE Transactions on Computers. pages 215-222, March 1981.

$\because \mathrm{H}-\mathrm{K}$ I. Ma. S. Devadas, A. R. Newton, and A. Sangiovanni-Vincentelli. Test generation for sequential circuits. In IEEE Transactions on $C A D$, pages 10811093. October 1988

10. II C. Paul and S. H. Unger. Minimizing the number of states in incompletely specified sequential circuits. In IRE Transactions on Electronic Computers, pages 35635\%. September 19.59

$11 !$ J. P. Roth. Diagnosis of automata failures: a calculus and a method. In $I B .11$ journal of Research and Development, pages 278-291. July 1966 